UICHEP-TH/92-15; FERMILAB-PUB-92/233-T; SLAC-PUB-5884

\title{
Novel Spin and Statistical Properties of Nonabelian Vortices
}

\author{
Lee Brekke $^{a}$, Hans Dykstra ${ }^{b}$, Adam F. Falk ${ }^{c}$, and Tom D. Imbo ${ }^{a}$
}

\begin{abstract}
We study the statistics of vortices which appear in $(2+1)$-dimensional spontaneously broken gauge theories, where a compact group $G$ breaks to a finite nonabelian subgroup $H$. Two simple models are presented. In the first, a quantum state which is symmetric under the interchange of a pair of indistinguishable vortices can be transformed into an antisymmetric state after the passage through the system of a third vortex with an appropriate $H$-flux element. Further, there exist states containing two indistinguishable spinless vortices which obey Fermi statistics. These results generalize to loops of nonabelian cosmic string in $3+1$ dimensions. In the second model, fractional analogues of the above behaviors occur. Also, composites of vortices in this theory may possess fractional "Cheshire spin" which can be changed by passing an additional vortex through the system.
\end{abstract}

${ }^{a}$ Department of Physics, University of Illinois at Chicago, Chicago IL 60607

${ }^{b}$ Fermi National Accelerator Laboratory, P. O. Box 500 Batavia IL 60510. Current Address: Department of Physics, University of Massachusetts at Amherst, Amherst MA 01003

${ }^{c}$ Stanford Linear Accelerator Center, Stanford CA 94309 
In $2+1$ dimensions, it is well known that when a compact gauge group $G$ is spontaneously broken via the Higgs mechanism to a finite subgroup $H$, there are topologically stable vortices labeled by $h \in H$ [1]. When $H$ is nonabelian, there is a long range interaction between these vortices due to a classical analog of the (nonabelian) Aharonov-Bohm effect [2] [3]. As a result, the classical and quantum mechanics of such multi-vortex systems is richer and more complex than that of their abelian counterparts. For example, the concept of indistinguishability of vortices is quite subtle and leads one to suspect that the statistical properties of these vortices may be extremely unusual. In this paper we consider a model where, in fact, the usual superselection rule between bosonic and fermionic statistics is violated. That is, a quantum state symmetric under the interchange of a pair of indistinguishable vortices can "mix" with an antisymmetric state. (The existence of such behavior requires the presence of one or more "spectator" vortices with appropriate $H$-flux elements.) While recently it has been recognized that it is possible to introduce such ambistatistical behavior into certain identical particle systems [4], in this theory it arises as a natural consequence of the long range vortex interactions. The above model also possesses states containing two indistinguishable spinless vortices obeying Fermi statistics, thus violating the usual spin-statistics relation. For systems of three or more indistinguishable vortices, parastatistical states [4] [5] also can occur, and in the presence of a spectator vortex different parastatistical types need not be superselected. We proceed to construct a second model which displays the fractional statistical analogue of each of the above behaviors. In this theory, there are also violations of the superselection rule between states with different values of $e^{2 \pi \mathrm{i} J}$, where $J$ is the total angular momentum. Our work may be considered as an application and extension of the ideas in [3], where a general framework is developed for studying vortex statistics.

We now recall some basic facts concerning the physics of nonabelian vortices. ${ }^{1}$ When internal excitations are ignored, a single vortex configuration is determined completely by the vortex position $x_{1} \in \mathbb{R}^{2}$ and its $H$-flux element $h$. This flux element may be thought of as the path-ordered exponential (or holonomy) of the corresponding gauge field around a closed contour, based at a fixed point $x_{0}$ at spatial infinity, which encircles the vortex once in a counterclockwise manner. For two vortices at $x_{1}$ and $x_{2}$, there are two fluxes $h_{1}$ and $h_{2}$ determined by path-ordered exponentials evaluated along contours $C_{1}$ and $C_{2}$.

1 In what follows, we work in temporal gauge $\left(A_{0}=0\right)$. Furthermore, we treat the vortices as pointlike objects and never allow the positions of any pair of them to coincide. 
We take $C_{i}$ to encircle the vortex at $x_{i}$ as shown in figure 1 . If the two vortices are moved along closed paths in $\mathbb{R}^{2}$, then when they return to their original positions their flux elements in general will have changed [2] [3]. In particular, if the vortex originally at $x_{1}$ encircles the one at $x_{2}$ once counterclockwise, then both fluxes are conjugated by the product $h_{1} h_{2}$. That is, in the final configuration the vortex at $x_{i}$ has flux $\left(h_{1} h_{2}\right) h_{i}\left(h_{1} h_{2}\right)^{-1}$. One way to see this is by transforming to a singular gauge in which the gauge potential is zero everywhere except on two strings beginning at $x_{1}$ and $x_{2}$ and stretching out to spatial infinity in different directions. As the $h_{1}$ vortex is brought around the $h_{2}$ vortex we deform the two strings so that they never intersect. ${ }^{2}$ In the final configuration these strings will have become entangled, and the above result may be obtained by recalculating the holonomies along $C_{1}$ and $C_{2}$. Note that these flux changes have a completely topological origin; at no point during the evolution has any vortex traversed a region of nonzero field. Similarly, if we exchange counterclockwise the positions of the two vortices with initial fluxes $h_{1}$ and $h_{2}$, then we end up with an $h_{1} h_{2} h_{1}^{-1}$ vortex at $x_{1}$ and an $h_{1}$ vortex at $x_{2}$. The above statements generalize in a natural way to a system of $N$ vortices. The total flux of an $N$-vortex system, defined as the path-ordered exponential along a counterclockwise contour which encircles all of the vortices, is conserved during the evolution of the system although the fluxes of individual vortices will in general become conjugated. If the vortices are located at the positions $x_{i}, 1 \leq i \leq N$, with fluxes $h_{i}$ (defined as in figure 1 ), then this total flux is $h_{1} h_{2} \cdots h_{N}$.

There is a subtle point concerning the distinguishability of vortices. Although two single-vortex fields with conjugate flux elements are distinct configurations in the broken gauge theory, they cannot be distinguished by any physical measurement. The standard procedure for probing the flux element of a vortex is by an Aharonov-Bohm scattering process involving particles carrying nontrivial $H$-charge. More precisely, suppose we take such a particle once around a vortex of flux $h$. If we assume that the particle is initially in a state $|u\rangle$ which transforms according to a faithful representation $D$ of $H$, then upon its return it will be in the state $D(h)|u\rangle$. We may then measure the matrix elements $\langle v|D(h)| u\rangle$ by interfering this particle with a variety of other particles in states $|v\rangle$ also transforming under $D$. In principle, it seems as though we may in this way determine all matrix elements of $D(h)$, and hence $h$ itself. However it has been shown that only

2 Note that in order for this path to be of finite action, the endpoints at spatial infinity of the two strings must remain fixed. 
the character $\chi^{(D)}(h)=\sum_{v}\langle v|D(h)| v\rangle$ can actually be obtained in this fashion, if one takes into account the effects of the creation and annihilation of virtual vortex-antivortex pairs [6]. Since $\chi^{(D)}\left(h_{1}\right)=\chi^{(D)}\left(h_{2}\right)$ if $h_{1}$ and $h_{2}$ are conjugate, we see that vortices with conjugate flux elements are indistinguishable. We note that this result holds even at energies well below the threshold for the creation of a real vortex-antivortex pair.

From the above discussion it is clear that vortices possessing conjugate flux elements should be considered as different internal states of a single species $\alpha$. One consequence of this for the quantum mechanics of such objects is that an isolated pair of vortices of type $\alpha$ need not be bosons. Indeed, they can be anyons. We will also see that in the presence of a third (distinguishable) vortex whose flux does not commute with a representative flux from the $\alpha$ species, the statistical behavior can be even more bizarre. In general, a quantum mechanical $N$-vortex state containing $n_{i}$ vortices of type $\alpha_{i}, 1 \leq i \leq k$, is a linear combination of states in which the vortices have well-defined fluxes. (We assume that the vortices are localized about the positions $x_{1}, \ldots x_{N}$ as in figure 1 , the first $n_{1}$ of these positions being associated with species $\alpha_{1}$, the next $n_{2}$ with species $\alpha_{2}$, etc.) These states may be obtained from each other by moving the vortices around closed loops in the plane and/or permuting the positions of vortices of the same species. We will denote by $\left|h_{1}, \ldots, h_{n_{1}} ; h_{n_{1}+1}, \ldots, h_{n_{1}+n_{2}} ; \ldots h_{N}\right\rangle$ the term in this sum in which the vortex localized about $x_{i}$ has flux $h_{i}$. Note that if we let $N_{i}=\sum_{j=1}^{i} n_{j}$, then the fluxes $h_{N_{i}+1}$ through $h_{N_{i+1}}$, for any fixed $0 \leq i \leq k$ (where $N_{0} \equiv 0$ ), must all lie in the same conjugacy class.

The loop and permutation operations in the above $N$-vortex system form a group known as the "partially colored braid group" $B_{n_{1}, \ldots, n_{k}}$ [7]. It can be interpreted as the fundamental group of the configuration space of our effective $N$-vortex system. The Hilbert space spanned by all possible states $\left|h_{1}, \ldots, h_{n_{1}} ; h_{n_{1}+1}, \ldots, h_{n_{1}+n_{2}} ; \ldots h_{N}\right\rangle$ decomposes into a direct sum of subspaces, each of which transforms according to an irreducible unitary representation (IUR) of $B_{n_{1}, \ldots, n_{k}}$. In each of these sectors, the statistics of the vortices of type $\alpha_{i}$ is determined by the action of the $\alpha_{i}$ exchange operations on the states. The group $B_{1,1}$, which is relevant for a system consisting of two distinguishable vortices, is isomorphic to the integers $\mathbb{Z}$, whose generator $\ell$ is taken to be the operation of circling the vortex at $x_{1}$ counterclockwise around the one at $x_{2}$. The action of $\ell$ on the corresponding two-vortex states is given by $\ell\left|h_{1} ; h_{2}\right\rangle=\left|g h_{1} g^{-1} ; g h_{2} g^{-1}\right\rangle$, where $g=h_{1} h_{2}$. The group $B_{2}$ which arises in a system of two indistinguishable vortices is again isomorphic to $\mathbb{Z}$, but this time the generator $\sigma$ is the operation which exchanges the two vortices in a counterclockwise manner. It acts as $\sigma\left|h_{1}, h_{2}\right\rangle=\left|h_{1} h_{2} h_{1}^{-1}, h_{1}\right\rangle$. As a final example, the 
group $B_{1,2}$ is an infinite discrete group which can be generated by two elements $\sigma$ and $\ell$, representatives of which are shown in figure 2 , subject to the single defining relation $(\sigma \ell)^{2}=(\ell \sigma)^{2}$. The action of these generators on the three-vortex states is given by $\ell\left|h_{1} ; h_{2}, h_{3}\right\rangle=\left|g h_{1} g^{-1} ; g h_{2} g^{-1}, h_{3}\right\rangle$ and $\sigma\left|h_{1} ; h_{2}, h_{3}\right\rangle=\left|h_{1} ; h_{2} h_{3} h_{2}^{-1}, h_{2}\right\rangle$, where $g=h_{1} h_{2}$. Since this group is nonabelian it has IUR's of dimension greater than one, which will lead to exotic statistical behavior in the associated Hilbert space sectors.

We will now illustrate these features of multi-vortex systems with two simple examples. In the first model the group $G=S U(2)$ is broken down to the eight element quaternion group $H=Q_{8}[8]$. The elements of $Q_{8} \subset S U(2)$ are \pm 1 and the Pauli matrices $a_{j}= \pm \mathrm{i} \tau_{j}$, $j=1,2,3$. There are five conjugacy classes, given by $\{1\},\{-1\}$ and $\left\{ \pm a_{j}\right\}$. We start with the state $\left|a_{3},-a_{3}\right\rangle$ containing two vortices of a single species $\beta$. The total flux of the system is trivial. A basis for a representation of the braid group $B_{2}$ can be obtained from the states $\sigma^{m}\left|a_{3},-a_{3}\right\rangle$ for integer $m$. Even powers of $\sigma$ operate trivially on this state, while odd powers yield $\left|-a_{3}, a_{3}\right\rangle \cdot{ }^{3}$ This two-dimensional representation of $B_{2}$ can be decomposed into a direct sum of two one-dimensional representations by forming the symmetric and antisymmetric combinations of the above states. In the corresponding Hilbert space sectors, the $\beta$ vortices are bosons and fermions respectively. The appearance of Fermi statistics is somewhat surprising since all of the fundamental fields in our theory are bosonic. (We will return to this point later.) If we start with a state of three or more $\beta$ vortices, not all of the same flux, then a similar procedure will also yield parastatistical Hilbert space sectors.

Next, consider the three-vortex state $\left|a_{1} ; a_{3}, a_{3}\right\rangle$ in which a single type $\alpha$ vortex (at $x_{1}$ ) has flux $a_{1}$, and two type $\beta$ vortices (at $x_{2}$ and $x_{3}$ ) have flux $a_{3}$. The total flux of the system is $-a_{1}$. We obtain a basis for a representation of the group $B_{1,2}$ by operating on this state with all combinations of the generators $\sigma$ and $\ell$ (and their inverses). This basis

3 By allowing the action of $\sigma$ on $\left|a_{3},-a_{3}\right\rangle$ to include a phase, it might seem that $\left|a_{3},-a_{3}\right\rangle$ and $\sigma^{2}\left|a_{3},-a_{3}\right\rangle$ need only be equal up to a phase. However, here $\sigma^{2}$ (unlike $\sigma$ ) can be thought of as a loop in the full gauge theory configuration space. The phase difference between the above two states is then just a "Berry's phase" with respect to this loop. But the gauge theory configuration space does not possess the appropriate topology for the existence of such nontrivial phases. Thus the above phase is 1 . Similar comments hold for the other examples in this paper. 
is

$$
\begin{aligned}
& \left|a_{1} ; a_{3}, a_{3}\right\rangle, \\
& \left|-a_{1} ;-a_{3}, a_{3}\right\rangle=\ell\left|a_{1} ; a_{3}, a_{3}\right\rangle, \\
& \left|-a_{1} ; a_{3},-a_{3}\right\rangle=\sigma \ell\left|a_{1} ; a_{3}, a_{3}\right\rangle, \\
& \left|a_{1} ;-a_{3},-a_{3}\right\rangle=\ell \sigma \ell\left|a_{1} ; a_{3}, a_{3}\right\rangle .
\end{aligned}
$$

Operating further with $\sigma$ or $\ell$ simply permutes these four states. This four-dimensional representation of $B_{1,2}$ reduces to a direct sum of two one-dimensional IUR's and one twodimensional IUR. This can be seen in the transformed basis

$$
\begin{aligned}
& |\psi\rangle=\frac{1}{2}\left(\left|a_{1} ; a_{3}, a_{3}\right\rangle+\left|a_{1} ;-a_{3},-a_{3}\right\rangle+\left|-a_{1} ;-a_{3}, a_{3}\right\rangle+\left|-a_{1} ; a_{3},-a_{3}\right\rangle\right), \\
& |\xi\rangle=\frac{1}{2}\left(\left|a_{1} ; a_{3}, a_{3}\right\rangle+\left|a_{1} ;-a_{3},-a_{3}\right\rangle-\left|-a_{1} ;-a_{3}, a_{3}\right\rangle-\left|-a_{1} ; a_{3},-a_{3}\right\rangle\right), \\
& \left|\phi_{1}\right\rangle=\frac{1}{\sqrt{2}}\left(\left|a_{1} ; a_{3}, a_{3}\right\rangle-\left|a_{1} ;-a_{3},-a_{3}\right\rangle\right), \\
& \left|\phi_{2}\right\rangle=\frac{1}{\sqrt{2}}\left(\left|-a_{1} ;-a_{3}, a_{3}\right\rangle-\left|-a_{1} ; a_{3},-a_{3}\right\rangle\right) .
\end{aligned}
$$

The vectors $|\psi\rangle$ and $|\xi\rangle$ carry one-dimensional IUR's $\rho_{1}$ and $\rho_{2}$ respectively, while $\left|\phi_{1}\right\rangle$ and $\left|\phi_{2}\right\rangle$ form a basis for a two-dimensional IUR $\rho_{3}$. The generators $\sigma$ and $\ell$ in these representations are

$$
\begin{array}{lll}
\rho_{1}(\sigma)=1, & \rho_{1}(\ell)=1, & \\
\rho_{2}(\sigma)=1, & \rho_{2}(\ell)=-1, \\
\rho_{3}(\sigma)=\left(\begin{array}{cc}
1 & 0 \\
0 & -1
\end{array}\right), & \rho_{3}(\ell)=\left(\begin{array}{ll}
0 & 1 \\
1 & 0
\end{array}\right) .
\end{array}
$$

In the sectors corresponding to $|\psi\rangle$ and $|\xi\rangle$, the vortices of type $\beta$ are bosons, since $\rho_{1}(\sigma)=$ $\rho_{2}(\sigma)=1$. In the $\rho_{3}$ sector they obey ambistatistics, which is neither Bose nor Fermi but contains aspects of each [4]].

It is interesting to see what happens to the states $\left|h_{1} ; h_{2}, h_{3}\right\rangle$ when we remove one of the vortices from the system. First, we note that the resulting two-vortex state will depend on the manner in which the third vortex is removed. That is, there is a variety of ways in which this vortex may be threaded through the others as it is dragged off to spatial infinity, and the total flux of the remaining two-vortex system will depend on the choice of this path [3]. If we choose to drag the vortex at $x_{3}$ in figure 2 off to the right, 
then $\left|h_{1} ; h_{2}, h_{3}\right\rangle$ will be reduced to the two-vortex state $\left|h_{1} ; h_{2}\right\rangle$. Applying this procedure to the states in (2), we find

$$
\begin{aligned}
& |\psi\rangle \rightarrow \frac{1}{\sqrt{2}}\left(\left|\psi_{+}\right\rangle+\left|\psi_{-}\right\rangle\right)=\frac{1}{2}\left(\left|a_{1} ; a_{3}\right\rangle+\left|-a_{1} ;-a_{3}\right\rangle\right)+\frac{1}{2}\left(\left|a_{1} ;-a_{3}\right\rangle+\left|-a_{1} ; a_{3}\right\rangle\right), \\
& |\xi\rangle \rightarrow \frac{1}{\sqrt{2}}\left(\left|\xi_{+}\right\rangle+\left|\xi_{-}\right\rangle\right)=\frac{1}{2}\left(\left|a_{1} ; a_{3}\right\rangle-\left|-a_{1} ;-a_{3}\right\rangle\right)+\frac{1}{2}\left(\left|a_{1} ;-a_{3}\right\rangle-\left|-a_{1} ; a_{3}\right\rangle\right), \\
& \left|\phi_{1}\right\rangle \rightarrow \frac{1}{2}\left(\left|\psi_{+}\right\rangle-\left|\psi_{-}\right\rangle+\left|\xi_{+}\right\rangle-\left|\xi_{-}\right\rangle\right), \\
& \left|\phi_{2}\right\rangle \rightarrow \frac{1}{2}\left(\left|\psi_{+}\right\rangle-\left|\psi_{-}\right\rangle-\left|\xi_{+}\right\rangle+\left|\xi_{-}\right\rangle\right) .
\end{aligned}
$$

Note that the two-vortex states $\left|\psi_{ \pm}\right\rangle$and $\left|\xi_{ \pm}\right\rangle$have total flux $\pm a_{3} a_{1}$. Each of these four states defines a one-dimensional IUR of the two-vortex braid group $B_{1,1}=\mathbb{Z}$ generated by the single loop $\ell$ in figure $2 ; \ell$ operates trivially on $\left|\psi_{ \pm}\right\rangle$and multiplies $\left|\xi_{ \pm}\right\rangle$by -1 . Thus the total Hilbert space breaks up into a direct sum of four pieces, each of which corresponds to one of these representations and has a fixed total flux. In fact, all twovortex states with a type $\alpha$ vortex at $x_{1}$ and a type $\beta$ vortex at $x_{2}$ appear here. Similarly, we can reduce the three-vortex states in (2) by removing the $\alpha$ vortex at $x_{1}$ off to the left, in which case we again obtain a basis for all two-vortex states (both being of type $\beta$ now). Note that $|\psi\rangle,|\xi\rangle$ and $\left|\phi_{1}\right\rangle$ yield bosonic two-vortex states, while $\left|\phi_{2}\right\rangle$ gives a fermionic state. In turn, it is straightforward to verify that all three-vortex states (including the ambistatistical ones in (2)) may be obtained by removing vortices from a larger system. In such a system, other superselection rules may be violated, such as that between Bose statistics and parastatistics, or between different forms of parastatistics.

The above analysis can be used to shed light on the nature of ambistatistics in this model. Consider an $a_{1}$ vortex which is incident from the left on the symmetric two-vortex state $\left(\left|a_{3}, a_{3}\right\rangle-\left|-a_{3},-a_{3}\right\rangle\right)$, and eventually combines with it to make the total state $\left|\phi_{1}\right\rangle$. If the $a_{1}$ vortex then loops around one (say the leftmost) of the other vortices, then $\left|\phi_{1}\right\rangle$ will become $\left|\phi_{2}\right\rangle$. So if we now remove the $a_{1}$ vortex as it came, the two $a_{3}$ vortices will be left in the anti-symmetric state $\left(\left|-a_{3}, a_{3}\right\rangle-\left|a_{3},-a_{3}\right\rangle\right)$. More generally, in the quantum mechanical scattering of an $a_{1}$ vortex off of the above bosonic state, this path along with many others will contribute. After the incident vortex has been scattered off to infinity, we will be left with a nonzero probability that the two remaining indistinguishable vortices are fermions. In this sense, ambistatistics violates the usual superselection rule between bosons and fermions. We stress that here this behavior is a natural consequence of the physics of nonabelian vortices, and has not been introduced in an ad hoc manner. 
We now turn to a model where the group $G=S U(3)$ is broken down to the six-element group $S_{3}$, the permutation group on three objects, which is generated by the matrices

$$
t_{1}=\left(\begin{array}{ccc}
0 & 1 & 0 \\
1 & 0 & 0 \\
0 & 0 & -1
\end{array}\right), \quad t_{2}=\left(\begin{array}{ccc}
-1 & 0 & 0 \\
0 & 0 & 1 \\
0 & 1 & 0
\end{array}\right)
$$

$S_{3}$ has three conjugacy classes, given by $\{1\},\left\{t_{1}, t_{2}, t_{1} t_{2} t_{1}\right\}$ and $\left\{t_{1} t_{2}, t_{2} t_{1}\right\}$. We start with the state $\left|t_{1}, t_{2}\right\rangle$, which contains two indistinguishable vortices since the fluxes $t_{1}$ and $t_{2}$ are conjugate. The total flux of the system is $t_{1} t_{2}$. We obtain a basis for a representation of the braid group $B_{2}$ by operating on this state with all powers of the vortex exchange operator $\sigma$. This basis is

$$
\begin{aligned}
& \left|t_{1}, t_{2}\right\rangle, \\
& \left|t_{1} t_{2} t_{1}, t_{1}\right\rangle=\sigma\left|t_{1}, t_{2}\right\rangle, \\
& \left|t_{2}, t_{1} t_{2} t_{1}\right\rangle=\sigma^{2}\left|t_{1}, t_{2}\right\rangle .
\end{aligned}
$$

Operating once more with $\sigma$ brings us back to the original state. This three-dimensional representation of $B_{2}$ decomposes into a direct sum of three one-dimensional representations. This can be seen by transforming to the basis

$$
\begin{aligned}
& \left|\lambda_{0}\right\rangle=\frac{1}{\sqrt{3}}\left(\left|t_{1}, t_{2}\right\rangle+\left|t_{1} t_{2} t_{1}, t_{1}\right\rangle+\left|t_{2}, t_{1} t_{2} t_{1}\right\rangle\right), \\
& \left|\lambda_{1}\right\rangle=\frac{1}{\sqrt{3}}\left(\left|t_{1}, t_{2}\right\rangle+\omega^{2}\left|t_{1} t_{2} t_{1}, t_{1}\right\rangle+\omega\left|t_{2}, t_{1} t_{2} t_{1}\right\rangle\right), \\
& \left|\lambda_{2}\right\rangle=\frac{1}{\sqrt{3}}\left(\left|t_{1}, t_{2}\right\rangle+\omega\left|t_{1} t_{2} t_{1}, t_{1}\right\rangle+\omega^{2}\left|t_{2}, t_{1} t_{2} t_{1}\right\rangle\right),
\end{aligned}
$$

where $\omega=\exp (2 \pi \mathrm{i} / 3)$. Each of the above states carries a one-dimensional representation of $B_{2}$ given by $\sigma\left|\lambda_{m}\right\rangle=\omega^{m}\left|\lambda_{m}\right\rangle, m=0,1,2$. In the Hilbert space sector spanned by $\left|\lambda_{0}\right\rangle$ the two indistinguishable vortices are bosons. In the $\left|\lambda_{1}\right\rangle$ and $\left|\lambda_{2}\right\rangle$ sectors they obey fractional statistics with statistical angles $\theta_{1}=2 \pi / 3$ and $\theta_{2}=4 \pi / 3$ respectively. Once again, this is somewhat surprising since all of the fundamental fields in our theory are bosonic. If we add one or more additional vortices from the $t_{1}$ conjugacy class to the above system, we will also obtain parastatistical sectors as well as their fractional generalizations (nonabelian anyons).

To help us understand these results, it is useful to introduce the notion of Cheshire charge. In a vortex system with total flux $h$, the only globally well-defined gauge transformations are those belonging to the subgroup $C_{H}(h) \subseteq H$ consisting of all elements that 
commute with $h$. States of the system may carry a nontrivial representation of $C_{H}(h)$, although this "Cheshire" charge is a global property of these states and is not carried by any individual vortex [8] 9]. Since the gauge transformations in $C_{H}(h)$ commute with elements of the appropriate braid group, we can choose states which simultaneously carry a representation of both groups. For the states (7) in the $S_{3}$ model, the globally well-defined subgroup $C_{S_{3}}\left(t_{1} t_{2}\right)$ is generated by $t_{1} t_{2}$ and is isomorphic to the cyclic group $\mathbb{Z}_{3}$. The state $\left|\lambda_{0}\right\rangle$ transforms trivially under this $\mathbb{Z}_{3}$, while $\left|\lambda_{1}\right\rangle$ and $\left|\lambda_{2}\right\rangle$ pick up the phases $\omega^{2}$ and $\omega$ respectively under a gauge transformation by $t_{1} t_{2}$. Thus, these anyonic states carry a nontrivial $\mathbb{Z}_{3}$ charge. The existence of this charge can shed some light on the emergence of fractional statistics in this example. Viewed from far away, these two-vortex systems look like composites of a point vortex with flux $t_{1} t_{2}$ and a nontrivial $\mathbb{Z}_{3}$ charge. Rotating this composite by $2 \pi$ (counterclockwise) has the same effect as performing a $t_{1} t_{2}$ gauge transformation. Thus the state $\left|\lambda_{1}\right\rangle$ (for instance), which picks up the phase $\omega^{2} \equiv e^{2 \pi \mathrm{i} J}$, has total angular momentum $J=2 / 3+$ integer. The fractional part of $J$ may be called "Cheshire spin" since it is a direct consequence of the Cheshire charge in the composite system and inherits similar nonlocal properties. From the point of view of the two original indistinguishable vortices which are contained in this "charged flux tube", there are three contributions to $J[7]$. The first two are the individual spins of the constituent vortices themselves. Since they are pure flux tubes, these contributions vanish. Finally, there is a contribution from one vortex encircling the other counterclockwise. This must give the entire spin of the system. However, this is the same as performing the double exchange operation $\sigma^{2}$ on $\left|\lambda_{1}\right\rangle$. Thus, a single exchange of the two constituent vortices must yield a phase $\pm e^{\pi \mathrm{i} J}$. Our earlier analysis shows that the plus sign is chosen. Note that the constituent vortices are spinless particles obeying fractional statistics, that is, they violate the usual spin-statistics relation. ${ }^{4}$ This result can be traced directly to the nonabelian nature of the vortices and the corresponding nonlocal interactions between them.

Systems with Cheshire behavior may violate the superselection rule between states with different statistical angles, as well as between states with different values of $e^{2 \pi \mathrm{i} J}$. For example, consider the passage of a vortex of flux $t_{1} t_{2}$ between the two vortices in the

4 Cheshire charge is also responsible for the emergence of Fermi statistics in the $Q_{8}$ model. Moreover, for the states in (2) the globally well-defined subgroup $C_{Q_{8}}\left(-a_{1}\right)$ is generated by $a_{1}$ and is isomorphic to the cyclic group $\mathbb{Z}_{4}$. The states $|\psi\rangle$ and $|\xi\rangle$ transform trivially under this $\mathbb{Z}_{4}$, while $\left|\phi_{1}\right\rangle$ and $\left|\phi_{2}\right\rangle$ change sign under a gauge transformation by $a_{1}$. Thus the ambistatistical sector of the Hilbert space carries a nontrivial $\mathbb{Z}_{4}$ Cheshire charge. 
state $\left|\lambda_{1}\right\rangle$. It is straightforward to show that in the resulting state we have $\sigma=1$ (the two constituent vortices are now bosons) and $e^{2 \pi \mathrm{i} J}=1$ (the composite now has integral angular momentum). The missing fractional spin is carried away as orbital angular momentum by the incident vortex as it moves off to infinity. In general, the passage of a vortex with a noncommuting flux from a distinct species can also change a nonabelian anyonic state into a state with different statistical properties.

Throughout this paper we have considered systems in which the number of vortices is constant, and we have neglected the creation of real vortex-antivortex pairs. It is reasonable to ask whether such processes impose constraints on the allowed multi-vortex states, and thereby possibly rule out certain types of exotic statistical behavior. Indeed, recent studies have indicated that only Bose, Fermi and fractional statistics are allowed for identical particles in the presence of pair creation, and furthermore that the usual spin-statistics relation holds for such particles [10]. However this work assumes that the particles carry no internal label. Hence it does not apply to parastatistical systems, for example, where it is known that consistent relativistic quantum field theories can be formulated [5]. The nonabelian vortex systems discussed here also avoid such restrictions, since two vortices of the same species can have distinct fluxes. Thus we believe that our results concerning the exotic statistics of vortices are not an artifact of our quantum mechanical approach.

We close with two brief comments. First, an analysis similar to that presented here may be applied to systems with other symmetry breaking schemes $G \rightarrow H$. While such a treatment generally will be more cumbersome, the emergence of exotic statistics for indistinguishable nonabelian vortices is generic. More precisely, an isolated system of indistinguishable vortices having noncommuting flux elements generally will possess anyonic states (possibly even nonabelian anyonic states if there are more than two vortices). States containing indistinguishable vortices in the presence of a spectator vortex with a noncommuting flux generally will violate the superselection rules between the above statistical behaviors, as in ambistatistical systems. Which representations of the appropriate braid group $B_{n_{1}, \ldots, n_{k}}$ are actually realized depends on the symmetry breaking scheme. Second, it is interesting to note that nonfractional exotic behaviors such as ambistatistics and parastatistics are in no way peculiar to $2+1$ dimensions. Similar results hold for closed loops of nonabelian cosmic string in the analogous $(3+1)$-dimensional spontaneously broken gauge theories. Here, the analogue of bringing one vortex around another is threading one loop of cosmic string through another loop. 
We would like to thank John March-Russell for helpful comments and interesting discussions. This work was supported in part by DOE contracts DE-FG02-84ER40173 (L. B. and T. I.) and DE-AC03-76SF00515 (A. F.). Most of this work was done while T. I. was a Junior Fellow in the Harvard Society of Fellows. 


\section{References}

[1] J. Preskill, in Architecture of the Fundamental Interactions at Short Distances, edited by P. Ramond and R. Stora (North-Holland, Amsterdam, 1987).

[2] F. Wilczek and Y.-S. Wu, Phys. Rev. Lett. 65 (1990) 13.

[3] M. Bucher, Nucl. Phys. B350 (1991) 163.

[4] T. D. Imbo, C. Shah Imbo and E. C. G. Sudarshan, Phys. Lett. B234 (1990) 103;

T. D. Imbo and J. March-Russell, Phys. Lett. B252 (1990) 84.

[5] Y. Ohnuki and S. Kamefuchi, Quantum Field Theory and Parastatistics (SpringerVerlag, Berlin, 1982);

A. P. Balachandran, Syracuse University preprint SU-4428-454 (1990), and references therein.

[6] M. G. Alford, K.-M. Lee, J. March-Russell and J. Preskill, Princeton University preprint PUPT-91-1288 (1991).

[7] L. Brekke, A. F. Falk, S. J. Hughes and T. D. Imbo, Phys. Lett. B271 (1991) 73.

[8] J. Preskill and L. M. Krauss, Nucl. Phys. B341 (1990) 50.

[9] M. G. Alford, S. Coleman and J. March-Russell, Nucl. Phys. B351 (1991) 735.

[10] A. P. Balachandran, A. Daughton, Z.-C. Gu, G. Marmo, R. D. Sorkin and A. M. Srivastava, Mod. Phys. Lett. A5 (1990) 1575;

R. D. Tscheuschner, Int. J. Theor. Phys. 28 (1989) 1269. 